\title{
The misperception of rotary motion
}

Two principles for predicting the relative frequency of illusory reversals of rotating plane objects were derived and tested empirically. Ten objects, variously combining valid and confounding depth cues, were used. Predictions based on the principles were confirmed in every case. The results are offered as an improved explanation of the Ames trapezoid illusion and other illusions of rotary motion.

Adlebert Ames, Jr. (1951) devised a trapezoidal framework made to resemble a window sash viewed at an angle (see Fig. 1). When rotated on a vertical axis, this framework appears to oscillate, that is, to flap back and forth with the taller side always nearer the viewer. Ames explained this illusion as due to the fact that we have learned to interpret trapezoidal forms as rectangles. Doors, windows, walls, buildings, etc., most often cast a trapezoidal image on the retina, yet we perceive them as rectangular. In other words, we exhibit shape constancy, In the case of rectangles the apparently taller side is always nearer. With the rotating trapezoid, therefore, the taller side is consistently seen as nearer although it is actually further from the viewer during half of each revolution. There are at least two difficulties with this explanation. There is, first, the fact noted by Zuckerman (1953) that a $S$ can simultaneously report the shape of the rotating object as trapezoidal and its motion as oscillation. The second difficulty is that initially most observers see the trapezoid rotating, then alternately rotating or oscillating, and then as oscillating constantly. One would have to suppose that the shape of the object is perceived first as trapezoidal, then as alternating, and finally as rectangular.

Subsequent work on this type of illusion (Day \& Power, 1963; Mulholland, 1956; Murray, 1961; Pastore, 1952; Zegers, 1964) has been directed toward a variety of factors such as the effects of viewing distance, size and shape of objects, tilt of axis, and surface pattern. It seems fairly well agreed that the strength of the illusion increases with distance and decreases with object size and with tilt of axis toward the S. Also, it is clear that the illusion can occur with a wide variety of objects, squares, circles, elipses and irregular shapes. The Ames explanation is clearly not applicable to nontrapezoidal objects.

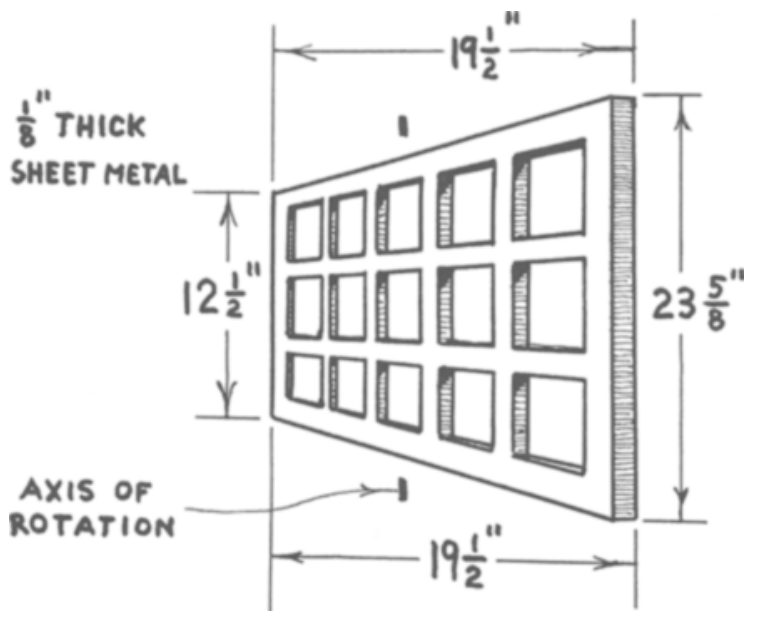

Fig. 1. The Ames trapezoidal window. This is a view of the trapezoid perpendicular to the line of sight. Both sides are painted alike, the frame white, the shadows grey, the panes cut out. When rotated the frame appears to oscillate. (After Ames, 1951.)

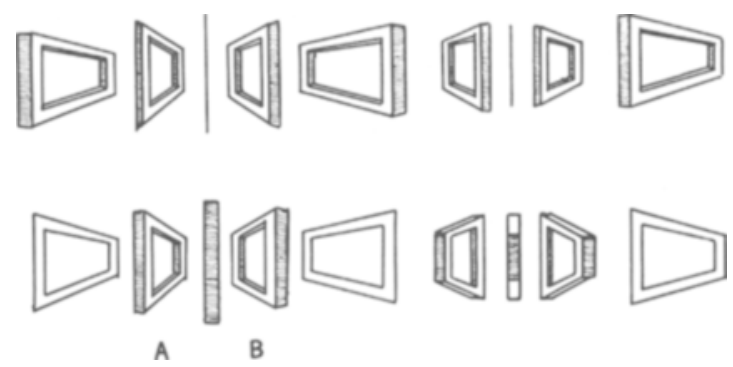

Fig. 2. The top row shows eight successive views of an artificially shadowed two-dimensional trapezoid in rotation. The bottom row shows the same views of a genuine three-dimensional trapezoid. Note the similarity between each view of the $2 \mathrm{D}$ trapezoid and the $A$ and $B$ views with the $3 D$ object. In the illusion, the $2 \mathrm{D}$ trapezoid appears with motion limited to the A-B portion of rotation.

Examination of available data suggests the hypothesis that the oscillatory illusion in rotating objects is not a misperception of motion per se but rather a faulty perception of depth. The parts of the objects appear to move from left to right, or right to left as they truly do. That is, the lateral component of their motion is perceived correctly. However, circular motion in a plane parallel to the line of sight cannot be perceived directly. Those components of the motion toward or away from the viewer must be mediated by depth cues.

Graham $(1963,1965$, 1968) has examined the role of the motion parallax cue (differential angular velocity) in the illusion. He points out that this cue provides ambiguous depth information and suggests that in the face of this ambiguity the cue of linear perspective is responsible for the illusion.

We agree with Graham that motion parallax is somewhat ambiguous in the rotating trapezoid, but we believe that it provides some true depth information. Figure 2 illustrates the cyclical expansion and contraction of both the long and short sides of trapezoids in rotation. Whenever viewing distance, object size, and illumination are adequate, this motion parallax information should support the veridical perception of rotary motion.

We agree further with Graham (1963) that linear perspective is a source of confounded depth information but contend that two other depth cues, interposition and shadow, also act to support the misperception of motion in the Ames trapezoid illusion.

The term interposition here refers to the cue which results from the physical fact that when light waves reflected to the eye from one object or surface are blocked by another object, the blocking object is closer. Unlike most other depth cues, the metric of the interposition cue is only ordinal, but it constitutes a strong cue to relative depth because of the stability of the physical facts from which it derives. The interposition simulated by painting facsimiles of the interior surfaces of a three-dimensional window frame on the surface of the Ames trapezoid results in these patches being seen during the entire revolution. In a genuine three-dimensional frame, these surfaces would be seen only half of each revolution as illustrated in Fig. 2. If the three-dimensional frame were really oscillated with one side always closer to the viewer, the interior surfaces would be seen during the entire motion. The fact of their always being visible in the two-dimensional Ames trapezoid, therefore, constitutes a strong cue to oscillatory motion.

Shadow is also employed in the Ames trapezoid to enhance the illusion. The patches painted to resemble the interior surfaces are done in a shade of gray while the surface resembling the exterior 
surfaces are white. These surfaces maintain constant relative brightness during the revolution much as they would during oscillation of a genuine three-dimensional frame.

Two general principles emerge from the foregoing analysis: (1) more illusory reversals can be expected with reduction in strength and number of true depth cues such as occurs with smaller objects and increased viewing distance; (2) more illusory motion can be expected with the increase in strength and number of misleading depth cues. The experiment reported here was designed (1) to test these theoretical principles empirically, (2) to investigate the relative strength of false shadow-interposition cues and false linear perspective cues, and (3) to explore the interaction between mutually antagonistic false depth cues.

\section{Stimulus Objects}

\section{METHOD}

Both rectilinear and circular stimulus objects were used as seen in Fig. 3. The objects were subdivided into five classes: (1) objects without confounding depth cues, (2) objects with shadowinterposition cues, (3) objects with linear perspective cues, (4) objects with shadow-interposition and perspective cues mutually compatible, and (5) objects in which shadow-interposition cues were incompatible with the perspective cues. The objects were cut from white index cards. Shadowing was done with black ink and the central area of each object was cut out. Object diam was 2 in.

The stimulus objects were mounted on a vertical shaft attached to a phonograph turntable. Illumination was provided by a $25-\mathrm{W}$ tungsten filament bulb placed $1 \mathrm{ft}$ above and behind the object. The objects were viewed through a circular aperture so that only the object was visible against a grey background. The viewing distance was $10 \mathrm{ft}$.

\section{Procedure}

The Ss, five male and five female college students, were tested individually. Binocular viewing with no restrictions on fixation was used to provide natural viewing conditions. Ss were read the following instructions:

This experiment is a study in the perception of motion. An object will be placed in this apparatus. Your task will be to watch its motion carefully to see when it changes its direction of rotation (demonstrated by experimenter). Ten different objects will be viewed for $1 \frac{11 / 2}{\mathrm{~min}}$ each. During this time an object may be seen always rotating in the same direction, it may change direction of rotation one or more times, and it may change direction so rapidly as to not make a complete revolution but rather will oscillate like this (demonstrated). In order to record the motion you can use the telegraph key. Press it whenever direction of rotation changes. If any objects oscillate you will be pressing the key quite often recording the rapid changes from clockwise to counterclockwise motion. Don't worry if you miss a few reversals-just count as many as you can. I'll tell you when to stop counting. Any questions?

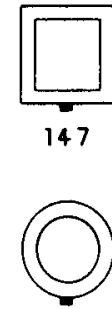

219

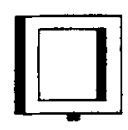

206

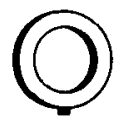

326

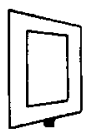

475

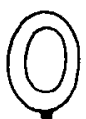

568

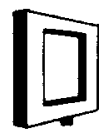

571

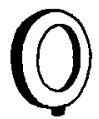

660

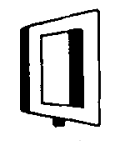

386

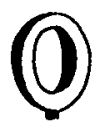

692
Fig. 3. The stimulus objects were patterned identically on both sides and rotated on a vertical shaft with the axis of rotation bisecting the figures. From left to right the objects incorporate: no confounding cues, shadow-interposition, linear perspective, shadow-interposition and perspective, and antagonistic confounding cues. The total number of illusory reversals of rotary motion reported, out of a possible 1000 , is shown for each object.
Table 1

Summary of an Analysis of Variance of Number of Illusory Reversals Reported by 10 Ss Viewing 10 Stimulus Objects

\begin{tabular}{lrrrr}
\hline Source & Sum of Squares & df & Mean Square & F \\
\hline objects & 34978.2 & 9 & 3886.5 & $6.46^{* *}$ \\
Order & 6065.6 & 9 & 674.0 & 1.12 \\
Individuals & 27531.8 & 9 & 3059.1 & $5.09^{* *}$ \\
Residual & 43303.4 & 72 & 601.4 & \\
Total & 111879.0 & 99 & & \\
\hline
\end{tabular}

$* * p<.01$

A 10 by 10 Latin square was used for ordering the presentation of the objects. Each S saw each object once, and each object was presented once in each ordinal position. The Ss' responses were recorded by an event marker on a kymograph drum. The viewing period for each object was $90 \mathrm{sec}$. At 33-1/3 rpm this allowed for a maximum of 100 illusory reversals in direction of rotation, two per revolution, for each $\mathrm{S}$.

\section{RESULTS}

Three a priori predictions were made, based on the analysis and general principles proposed above: (1) Shadowed and/or perspectived objects would be more productive of illusory reversals than comparable plane figures because they incorporate confounding depth cues; (2) shadowed and perspectived objects would be more productive of illusion than comparable figures incorporating either shadow or perspective alone; (3) circular objects would be more productive of illusion than rectilinear objects. This third prediction was based on the fact that circular objects provide less true depth information to counteract confounding cues then do rectilinear objects of comparable dimension.

Figure 3 contains the total number of illusory reversals recorded by the $10 \mathrm{Ss}$ for each of the stimulus objects. The ordering of these results is exactly as predicted above. Table 1 summarizes an analysis of variance performed on the data which indicates that the differences between the objects considered collectively are statistically significant.

While it is improbable that all of the predicted events occurred by chance, it is possible that some of them may have. Specific predictions were tested against the uncontrolled variability inherent in the data. Conventional multiple pair comparison techniques seemed inappropriate in that not all of the 45 potential pair comparisons possible between the means for the 10 stimulus objects were the object of prediction. In order to provide for significance tests of the predicted results while maintaining a reasonably low probability of Type II error, orthogonal comparisons between treatment sums were employed (Edwards, 1960).

The results of the orthogonal comparisons indicate that: (a) as predicted, shadowed and/or perspectived objects were more productive of illusory reversals than the comparable plain figure $(\mathrm{p}<.01)$, (b) as predicted, shadowed and perspectived objects were more productive of illusory reversals than comparable objects with shadowing or perspective alone $(p<.05)$, (c) as predicted, circular objects were more productive of illusory reversals than rectilinear objects $(\mathrm{p}<.01)$.

Comparison of confounding cues. No predictions were made concerning the relative effectiveness of artificial shadowing and perspective. The orthogonal tests of these relations indicate that for the objects used, perspective alone was significantly more productive of the illusion than shadowing alone $(p<.05)$. This result cannot be generalized beyond the specific objects used in this experiment since variation of the intensity and width of the artificial shadowing would probably be related to the strength of the effects of shadowing. Maximum illusion production presumably would occur when the painted shadow intensity is matched to real shadow intensity under the particular illumination conditions employed in a given experiment. Variation in the amount of perspective incorporated into an object is a!so possible, 
with focal distance and angle of presentation as independent variables contributing to the particular projection selected for the stimulus object. Further experimentation could probably determine the functional relations existing between these and other variables, such as rotation speed, viewing distance, object size, object-background brightness gradient, etc. The variables mentioned were held at constant values in the present experiment so that they should not affect the relations among the various stimulus groupings which were the object of theoretical predictions.

Interaction of opposed cues. The objects in which perspective and shadows gave mutually contradictory depth cues were included for exploratory purposes. The circular object produced 692 reversals, more than any other object, while the square object produced 186, ranking fifth among the 10 objects. The comparison of Ss after viewing and counting reversals of these objects suggested that the darkened edges were almost always seen as nearer the viewer during illusory oscillation. This finding suggests in turn that when in conflict, shadow-interposition cues take precedence over perspective cues. Again this inference may be correct only for the specific parameters of the cues used in this experiment, but it does seem reasonable to suspect that this result may be more general. The stability of interposition as a depth cue is virtually inviolate, while perspective is subject to a variety of size-distance interpretations. This is not to say that artificial shadows will be more readily taken to be depth indicators, but rather that when they are so taken they will manifest stronger depth determination than will perspective.

\section{DISCUSSION}

The Ames trapezoid illusion is a striking example of an entire class of illusions in which rotary motion is misperceived due to either a lack of sufficient valid depth cues or the presence of false depth cues or both. The windmill illusion, described as early as 1759 by W. Porterfield (Boring, 1942), the silhouetted fan blade studied by Miles (1931), and the rotating circles, squares, and what-have-you studied by Mulholland (1956), Murray (1961), and Day and Power (1963) all constitute further examples of this class of illusions. While only the most naive would suppose that a stick inserted in water was truly bent, or that a Necker cube or Schroeder staircase physically popped in and out from the paper, the case of a farmer (Miles, 1929) who forced a salesman to replace his windmill because it sometimes " $\operatorname{ran}$ in the wrong direction" gives evidence of the strength of illusions of the type considered here. Our own Ss all gave evidence of their belief that the reversals they reported actually occurred.

This experiment demonstrates the possibility of explanation of the oscillatory illusion in terms of stimulus factors rather than less testable organismic factors such as the "assumption of rectilinearity." Our proposed explanation seems able to encompass a fairly wide range of empirical data, to avoid several difficulties faced by previous authors, and to generate fairly detailed predictions which have been verified empirically. The results do not explain the ability of some Ss to cause voluntary reversals of apparent rotation or oscillation. Neither do they explain the tendency for practice to increase the strength of such illusions. This tendency is the more remarkable since it constitutes a rare example of learning to misperceive. How such learning is adaptive, if it indeed is adaptive, is not at all clear. We are attempting to examine the role of eye movements in these illusions. Our analyses suggest that less complex eye movements are involved in seeing oscillation than in seeing rotation and that such eye movements also result in a suppression of valid depth cues.

\section{REFERENCES}

AMES, A. Visual perception and the rotating trapezoidal window. Psychological Monographs: General \& Applied, 1951, 65, No. 7.

BORING, E. G. Sensation and perception in the history of experimental psychology. New York: Appleton-Century-Crofts, 1942.

DAY, R. H., \& POWER, R. P. Frequency of apparent reversal of rotary motion in depth as a function of shape and pattern. Australian Journal of Psychology, 1963, 15, 162-174.

EDWARDS, A. L. Experimental design in psychological research. (Rev. ed.) New York: Rinehart \& Co., 1960.

GRAHAM, C. H. On some aspects of real and apparent visual movement. Journal of the Optical Society of America, 1963, 53, 1015-1025.

GRAHAM, C. H. Perception of movement. In C. H. Graham (Ed.), Vision and visual perception. New York: Wiley, 1965.

GRAHAM, C. H. Depth and movement. American Psychologist, 1968, 23, 18.26.

MILES, W. R. Figure for the windmill illustration. Journal of General Psychology, 1929, 2, 143-144.

MILES, W. R. Movement interpretations of the silhouette of a revolving fan. American Journal of Psychology, 1931, 43, 392-405.

MULHOLLAND, T. B. Motion perceived while viewing rotating stimulus objects. American Journal of Psychology, 1956, 69, 96-99.

MURRAY, P. A. A quantitative study of the Ames oscillatory illusion. Dissertation Abstracts, 1961, 22, No. 11.

PASTORE, N. Some remarks on the Ames oscillatory effect. Psychological Review, 1952, 59, 319-323.

ZEGERS, R. T. The reversal ilusion of the Ames trapezoid. Transactions of the New York Academy of Sciences, 1964, 26, 377-400.

ZUCKERMAN, B. D. The phenomenal movement of the rotating trapezoidal window; an experimental investigation. Unpublished Master's thesis. New School for Social Research, 1953.

\section{NOTE}

1. Address: Department of Psychology, St. Louis University, St. Louis, Mo. 63103.

(Accepted for publication August 9, 1968. 